\title{
Juego patológico, patrones de personalidad y síndromes clínicos
}

\section{Pathological gambling, personality patterns and clinical syndromes}

\author{
Margarita Ortiz-Tallo*; Constanza Cancino*; \\ Sebastián Cobos ${ }^{* *}$
}

\author{
* Universidad de Málaga \\ ** Colaboradores del grupo de Investigación HUM 300 \\ Enviar correspondencia a: \\ Margarita Ortiz-Tallo. Dpto. de Personalidad, Evaluación y \\ Tratamiento Psicológico, Facultad de Psicología. \\ Boulevard Louis Pasteur, s/n. 29071 Málaga. \\ (34) 952132555, mortiztallo@uma.es
}

\section{RESUMEN}

El objetivo de este estudio es evaluar la presencia de patrones de personalidad patológicos y explorar su relación con los síndromes clínicos, en una muestra de 212 varones jugadores patológicos pertenecientes a un centro de rehabilitación. Asimismo explorar la posible existencia de perfiles patológicos, comunes en los jugadores, en los que puedan coexistir distintos trastornos de personalidad y síndromes clínicos. Se ha utilizado el Inventario Clínico Multiaxial de Millon (MCMI-II). Los resultados muestran cuatro patrones de personalidad clínicos de mayor frecuencia: dependiente, obsesivo-compulsivo, narcisista y antisocial. Los sindromes clínicos más frecuentes son el abuso de alcohol y el abuso de drogas. Se analiza la relación existente entre cada uno de los patrones de personalidad y los síndromes clinicos. Posteriormente se realiza un análisis de cluster y se identifican dos perfiles de personalidad diferenciados, con rasgos pertenecientes a distintas escalas, que se han denominado "perfil antisocial" y "perfil obsesivo compulsivo", cada uno de ellos se relaciona de manera muy diferente con los sindromes clínicos. Mostrando el grupo antisocial, de forma estadísticamente significativa, puntuaciones más altas en todos los síndromes clínicos, siendo cercanas a la puntuación clínica en abuso de alcohol, abuso de drogas, pensamiento psicótico y trastorno delirante.

Palabras clave: Juego patológico; trastornos de personalidad; síndromes clínicos.

\section{ABSTRACT}

The aim of this study is to evaluate the prevalence and percentages of personality patterns, and explore their relationship with clinical syndromes in a sample of 212 pathological gamblers from a rehabilitation center. The study also explores the possibility of profiles, common in gamblers, in which personality disorders and clinical syndromes coexist. The Millon Clinical Multiaxial Inventory (MCMIII) was employed. Four clinical personality patterns were the most frequently identified: dependent, obsessive-compulsive, narcissistic and antisocial. The relationship between personality patterns and clinical syndromes was also analyzed. Subsequent cluster analysis revealed two personality profiles, with features belonging to different scales, called "antisocial profile" and "obsessive-compulsive profile". These two profiles differ considerably in their relation to clinical syndromes. The "antisocial" group scores significantly higher on all clinical syndromes, and close to the clinical score in the cases of alcohol abuse, drug abuse, psychotic thinking and delusional disorder.

Keywords: Pathological gambling; personality disorders; clinical syndromes. 


\section{INTRODUCCIÓN}

$\mathrm{E}$ n los últimos años se ha mostrado un gran interés por analizar la existencia de una posible patología dual en las personas con adicciones, se ha tratado de valorar especialmente si a la adicción le acompaña un trastorno de personalidad. En el caso del juego patológico sería de interés estimar, por tanto, la comorbilidad con trastornos de la personalidad pero también es importante evaluar la coexistencia de otros sindromes clínicos, tales como el consumo de alcohol, de drogas, la depresión o la ansiedad. El conocimiento de ello aporta información relevante para el pronóstico y para el tratamiento de las personas con adicción al juego.

Una de las principales aportaciones del modelo de Theodore Millon a la psicopatología tiene que ver fundamentalmente con la naturaleza interactiva y multiaxial entre la personalidad y los síndromes clínicos (Cardenal, Sánchez y Ortiz-Tallo, 2007; Millon, 2006). Esta relación interactiva y multiaxial entre los síndromes clínicos y la personalidad (Eje I y Eje II del DSM, Diagnostic and Statistical Manual of Mental Disorders) (APA, 1980, 2000), Ileva a concluir que es la personalidad el lugar en el que se producen los comportamientos patológicos, de forma que la única manera de darle sentido al síndrome clínico, es conociendo y valorando la forma de ser de la persona que presenta la alteración.

Centrándonos en los sindromes clínicos del Eje I las investigaciones de Black y Moyer (1998) encuentran un 10\% de jugadores patológicos con problemas de abuso de sustancias y $10 \%$ de jugadores con consumo de alcohol. La adicción a sustancias en jugadores patológicos, se observa en una alta relación con los trastornos del estado de ánimo (Stewart y Kushne, 2003). Llinares, Santos, Camacho, Albiach, Palau, (2003), encuentran un 39,9\% de consumo exclusivo de alcohol asociado al juego. Otros autores (Krishnan y Orford, 2002), observan que el alcohol es un factor predictivo para los problemas del juego. Sin embargo, la comorbilidad de síndromes clínicos no indica necesariamente una relación de causalidad, ya que juego patológico y los problemas anteriormente mencionados, podrían situarse para algunos autores, tanto como riesgo de desarrollo de la ludopatía o como consecuencia de ésta (Estévez y Calvete, 2007; Slutske et al., 2005).

Algunas investigaciones destacan la existencia de una alta comorbilidad con los trastornos afectivos (Boughtton y Falenchuk, 2007; El-Guebaly et al, 2006). De hecho, se considera que existe hasta un 75\% de los pacientes hospitalizados con diagnóstico de juego patológico que cumplen criterios para un trastorno depresivo mayor, y $28 \%$ de éstos tienen episodios depresivos recurrentes (Bahamón, 2006). La manía y la hipomanía también se encuentran asociadas (Desai y Potenza, 2008).

Por otra parte, las investigaciones que han tratado de conocer la relación entre juego patológico y trastornos de personalidad (Eje II del DSM) han Ilegado a diversas conclusiones. Algunos autores (Blaszcynski, Steel y McConaghy, 1997; lbañez, et al, 2001; Pietrzak y Petry, 2005) han destacado el trastorno de personalidad antisocial junto a la ludopatía. El estudio Ilevado a cabo por Pietrzak y Petry (2005), encuentra una prevalencia de un 16,5\% de jugadores patológicos que cumplen los criterios diagnósticos para trastorno de personalidad antisocial, y un 20,5\% de estos, ha caído en actividades delictivas para mantener el juego. Mientras que Cunningham-Williams, Cottler, Compton y Spitznagel, (1998), hallan cifras mayores de un 35\% de jugadores patológicos que cumplen con el criterio antisocial de personalidad. Estos mismos autores posteriormente refieren un 22\% de jugadores patológicos con criterio antisocial que además mantienen problemas de uso de drogas (CunninghamWilliams et al., 2000). Bahamón (2006) indica que este perfil de jugadores no suele buscar ayuda y tratamiento para sus problemas con el juego, lo que podría suponer que tuvieran una menor presencia en la clínica.

El trastorno de personalidad obsesivo-compulsivo, también se encuentra en una alta relación con el juego patológico (Sacco, Cunningham-Williams, Ostmann, Spitznagel, 2007). Se observa en la revisión de Durdle y Stewart (2007) que el juego patológico compartiría la fenomenología, la etiología y aspectos como la edad de inicio (Kim y Grant, 2001).

Henderson (2004), en una muestra de pacientes psiquiátricos ambulatorios, administra el Inventario Clínico Multiaxial de Millon (MCMI-II), encontrándose que los únicos factores predictivos de tener problemas con el juego patológico, son la personalidad obsesivo-compulsiva y la evitativa.

Otros autores han asociado el juego patológico con la personalidad límite (Bagby, Vachon, Bulmash y Quilty, 2008; Blaszczensky et al.,1997; Blaszczensky y Steel, 1998).

Se sugiere que las personas con el diagnóstico dual, trastorno de personalidad y juego patológico, tienen más probabilidades de dedicarse a conductas adictivas como el alcohol o el uso de drogas (French, Maclean y Ettner, 2008; Melville, Casey, Kavanagh, 2007). Este hecho influiría en el pronóstico y evolución del tratamiento psicológico, presentando más dificultades para recuperarse de sus problemas de juego (Goodyear-Smith et al., 2006, Labrador, 2008).

Para evaluar el juego patológico se han utilizado fundamentalmente cuestionarios de autoinforme y entrevistas clínicas (Black y Moyer, 1998; Blaszcynski y Steel, 1998; Cunningham-Williams, Cottler, Compton, Spitznagel y BenAbdallah, 2000; Pietrzak y Petry, 2005; Slutske, Caspi, Moffit, Poulton, 2005). El inventario clínico multiaxial de Millon (MCMI-II,1998) ha sido usado para analizar la personalidad de los jugadores (Fernández-Montalvo y Echeburúa, 2006; Henderson, 2004; Pedrero Pérez, 2009). El MCMI de Millon, es un instrumento multiaxial derivado de un modelo integrado de la psicopatología y la personalidad, y a su vez, relacionado con el modelo multiaxial del DSM (Cardenal, Sánchez y Ortiz-Tallo, 2007).

Los estudios realizados en España destacan el interés en evaluar la posible patología dual de los jugadores patológicos con los trastornos de personalidad. Sin embargo, hay escasos estudios que exploren la relación con otros síndromes clínicos. Asimismo consideramos de interés indagar la posible existencia de perfiles de personalidad comunes entre los jugadores, en los que puedan coexistir distintos trastornos de personalidad y síndromes clínicos. 
Este trabajo forma parte, además, de una investigación más amplia que pretende conocer si hay patrones de personalidad o perfiles que predicen la rehabilitación o la recaída en el juego patológico. Para ello se está haciendo un seguimiento de tres años a este grupo evaluado al llegar al centro de rehabilitación de jugadores.

El objetivo del presente estudio es evaluar a jugadores patológicos pertenecientes a un centro de rehabilitación, a través del MCMI-II para:

1) Identificar la presencia de patrones de personalidad patológicos y de síndromes clínicos.

2) Explorar la relación entre los patrones de personalidad patológicos y los síndromes clínicos.

3) Explorar la presencia de perfiles de personalidad comunes (coexistencia de varios patrones de personalidad patológicos) y su relación con los síndromes clínicos.

\section{MÉTODO}

\section{Participantes}

El estudio se realiza en Málaga, en un principio se comienza con 235 personas, que acuden a tratamiento del juego patológico en una asociación de rehabilitación de juego patológico. La muestra que finalmente se presenta es de 212 varones. Se ha tomado esta decisión al estar representadas las mujeres solo por un grupo de 23.

Los criterios de inclusión en el estudio han sido: 1) haber acudido a la Asociación de Jugadores en rehabilitación para solicitar ayuda durante los años 2006 al 2008; 2) tener un diagnóstico de juego patológico valorado por el psicólogo del Centro; 3) encontrarse dentro de los 3 primeros meses de asistencia en la Asociación; 4) ser varón; y 5) haber cumplimentado de forma correcta el cuestionario MCMI-II. Los criterios de exclusión han sido: 1) ser mujer; y 2) perfiles inválidos en el MCMI-Il (considerados inválidos por la puntuación obtenida en los índices de Validez o de Sinceridad).

Las edades se encuentran entre los 20 y 72 años, correspondiendo a una media de 41 años aproximadamente, con una moda de 30 años. En lo que respecta al estado civil, el $58,5 \%$ se encuentran casados, $32,1 \%$ solteros y $9,4 \%$ divorciados. En relación al nivel de estudios, se observa un predominio de jugadores con estudios básicos $61,3 \%$, seguido de secundarios $26,4 \%$, universitarios $8,5 \%$ y sin estudios $3,8 \%$. La mayoría $(67,9 \%)$, son personas que desempeñan empleos relacionados con el sector servicios, un $16,5 \%$ son agentes comerciales, el 9,4\% asalariados y, por último, un 9,4\% están desempleados y un 2,8\% jubilados.

En cuanto al tiempo de juego, se observó una media de 10 años, siendo la moda de 2 años. Por otra parte, el 65,7\% refiere no consumir alcohol y $34,3 \%$ expresan tener problemas con éste.

\section{Instrumentos}

Se realiza una historia clínica general, diseñada por el Centro para todas las personas que acuden a solicitar ayuda y que contempla los aspectos relacionados con: sexo, edad, estado civil (casado, soltero y divorciado), escolaridad (estudios básicos, secundarios, universitarios y sin estudios), área de empleo (servicios, agentes comerciales, asalariados, desempleados y jubilados), cantidad de años de juego (número de años expresado) y consumo de alcohol expresado.

Con la finalidad de evaluar patrones de personalidad y conocer la prevalencia de síndromes clínicos, se utiliza el Inventario clínico multiaxial de Millon-II (MCMI-II; Millon, 1987) en la adaptación española de Ávila-Espada, Jiménez Gómez y colaboradores (1999), por ser la versión con la que se inició el proceso de evaluación, anterior a la adaptación del MCMI-III. EI MCMI-II (1999), constituye una escala de auto-informe con 175 ítems, donde cada uno es contestado en forma de afirmación o rechazo (V-F). Los ítems se encuentran configurados dentro de 22 escalas clínicas y tres escalas de corrección (sinceridad, deseabilidad y alteración) orientadas a identificar la tendencia de revelar, esconder o favorecer ciertas respuestas.

Las escalas de personalidad, corresponden en su mayoría al Eje II del DSM-IV, y son las siguientes: esquizoide, evitativa, dependiente, histriónica, narcisista, antisocial, agresivo/sádico, obsesivo-compulsivo, pasivo/agresivo, y autodestructivo y las siguientes escalas relacionadas con alteraciones graves de la personalidad: esquizotípico, límite y paranoide. Por último, las escalas relativas a los sindromes clínicos, relacionadas con el Eje I del DSM-IV, representan estados que pueden ser transitorios en un proceso patológico: ansiedad, histeriforme, hipomanía, neurosis depresiva, abuso de alcohol y abuso de drogas. Siendo consideradas de mayor gravedad las escalas: pensamiento psicótico, depresión mayor y trastorno delirante.

Para la evaluación de las escalas de personalidad se considera una puntuación de tasa base (TB) mayor de 75 como indicador de tendencia de la persona a patrones clínicos de personalidad y TB de 85 en adelante, indicaría trastorno de personalidad. La evaluación de los síndromes clínicos, considera un TB mayor de 75 para designar una puntuación clínica.

En la validación española el instrumento presenta criterios adecuados de fiabilidad y validez. Las puntuaciones de estabilidad arrojan correlaciones medias de 0.78 para las escalas de personalidad y de 0.80 para las escalas de síndromes clínicos (Avila-Espada, Jiménez Gómez y cols, 1999).

\section{Procedimiento}

Se ha realizado el estudio con pacientes que acudian a un programa de rehabilitación de ludopatía en el centro AMALAJER de la ciudad de Málaga, durante los años 2006 al 2008. Los datos fueron recogidos siempre por el mismo psicólogo, miembro del equipo de investigación.

La evaluación fue llevada a cabo de forma individual en un despacho y se les informó que los datos serían utilizados para llevar a cabo un estudio sobre el juego patológico. Se 
recogió la historia clínica general con los datos anteriormente mencionados y posteriormente, se administró el Inventario clínico multiaxial de Millon-II (MCMI-II), con el propósito de evaluar patrones de personalidad y síndromes clínicos.

\section{Análisis de datos}

Toda la información ha sido codificada y analizada mediante el programa estadístico SPSS ${ }^{\circledast} 17.0$ para Windows ${ }^{\oplus}$.

Para evaluar la presencia de patrones clínicos de personalidad y sindromes clínicos, se ha calculado la frecuencia de los mismos, tomando como punto de corte la puntuación de 75 TB, indicadora de patrón clínico de personalidad y de presencia de síndrome clínico.

Se han llevado a cabo correlaciones de Pearson entre los cuatro patrones clínicos de personalidad con mayor frecuencia y los síndromes clínicos con la finalidad de conocer la relación existente entre ellos.

El procedimiento utilizado para clasificar a los sujetos en perfiles comunes o grupos homogéneos ha sido el análisis de conglomerados mediante el método Ward (método de análisis jerárquico en el que la diferencia entre los grupos se calcula mediante la distancia euclídea al cuadrado). El análisis de realizó a partir de todas las puntuaciones en los patrones de personalidad. Por último, se han analizado las diferencias entre los dos perfiles comunes obtenidos en relación a los sindromes clínicos, para ello se ha llevado a cabo una prueba $\mathrm{t}$ para muestras independientes.

\section{RESULTADOS}

\section{Frecuencia de patrones clínicos de personalidad y síndromes clínicos}

Los resultados se presentan en las Tablas 1 y 2, mostrando que los patrones de personalidad con mayor frecuencia son los dependiente, obsesivo-compulsivo, antisocial y narcisista, y el de menor frecuencia el esquizoide. Asimismo, los síndromes clínicos más frecuentes son el abuso de alcohol y el abuso de drogas, y el menos frecuente la depresión mayor.

\section{Correlaciones entre los cuatro patrones clínicos de personalidad con mayor frecuencia y los síndromes clínicos}

Los resultados se presentan en la Tabla 3 y muestran que el patrón de personalidad dependiente se relaciona de forma positiva con ansiedad, hipomanía e histeriforme. El patrón obsesivo-compulsivo sólo se relaciona de forma positiva con el síndrome histeriforme. El patrón antisocial se asocia positivamente con abuso de alcohol, abuso de drogas, trastorno delirante y pensamiento psicótico. Finalmente, el patrón narcisista se relaciona también de forma positiva con abuso de drogas, trastorno delirante, hipomanía, pensamiento psicótico e histeriforme.
Tabla 1. Frecuencia y porcentaje de patrones clínicos de la personalidad

\begin{tabular}{lcc}
\hline \multicolumn{2}{c}{ TB>75 } \\
Patrones de Personalidad & $\%$ & N \\
\hline Patología moderada de la personalidad & & \\
\hline Dependiente & 29,7 & 63 \\
\hline Obsesivo-Compulsivo & 29,3 & 62 \\
\hline Antisocial & 23,6 & 50 \\
\hline Narcisista & 23,6 & 50 \\
\hline Agresivo-Sádico & 22,7 & 48 \\
\hline Pasivo-Agresivo & 22,7 & 48 \\
\hline Histriónico & 18,4 & 39 \\
\hline Autodestructivo & 18,4 & 39 \\
\hline Fóbico & 17,5 & 37 \\
\hline Esquizoide & 16,5 & 35 \\
\hline Patología severa de la personalidad & & \\
\hline Paranoide & 16,1 & 34 \\
\hline Limite & 12,3 & 26 \\
\hline Esquizotípico & 10,4 & 22 \\
\hline
\end{tabular}

Tabla 2. Frecuencia y porcentaje de síndromes clínicos

\begin{tabular}{lcc}
\hline & \multicolumn{2}{c}{ TB>75 } \\
Sindromes Clínicos & N & $\%$ \\
\hline Abuso de alcohol & 52 & 24,5 \\
\hline Abuso de drogas & 34 & 16 \\
\hline Ansiedad & 33 & 15,6 \\
\hline Trastorno delirante & 33 & 15,6 \\
\hline Hipomanía & 29 & 13,7 \\
\hline Pensamiento psicótico & 26 & 12,3 \\
\hline Neurosis depresiva & 21 & 9,9 \\
\hline Histeriforme & 20 & 9,4 \\
\hline Depresión mayor & 16 & 7,5 \\
\hline
\end{tabular}

Perfiles de patrones de personalidad comunes y diferencias en los grupos en los sindromes clínicos

Por otro lado, con objeto de comprobar la existencia de posibles perfiles de patrones de personalidad comunes en los jugadores patológicos, al realizar el análisis de conglomerados la inspección visual del dendograma sugiere una solución de dos grupos que es clínicamente interpretable. Las medias de los dos grupos en los patrones clínicos y los valores de las pruebas $t$ para muestras independientes se presentan en la Tabla 4. Los grupos se encuentran representados por 99 y 135 sujetos, respectivamente. El primer grupo obtiene pun- 
Tabla 3. Coeficiente de correlación de Pearson entre Patrones clínicos de Personalidad y Síndromes Clínicos

\begin{tabular}{lcccc} 
& & \multicolumn{2}{c}{ Patrones clínicos de personalidad } \\
Síndromes Clínicos & Dependiente & Antisocial & Obsesivo-Compulsivo & Narcisista \\
\hline Abuso de alcohol & .141 & $.309^{*}$ & -.112 & .214 \\
\hline Abuso de drogas & .181 & $.422^{* *}$ & .072 & $.389^{* *}$ \\
\hline Ansiedad & $.212^{*}$ & .004 & .065 & .359 \\
\hline Trastorno delirante & .190 & $.537^{* *}$ & .168 & $.389^{* *}$ \\
\hline Hipomanía & $.301^{* *}$ & .219 & .081 & $.510^{* *}$ \\
\hline Pensamiento psicótico & .027 & $.243^{*}$ & -.052 & $.301^{*}$ \\
\hline Neurosis depresiva & .159 & .078 & -.026 & .403 \\
\hline Histeriforme & $.214^{*}$ & .152 & $.239^{*}$ & $.257^{*}$ \\
\hline Depresión mayor & .181 & .152 & .053 & .223 \\
\hline
\end{tabular}

${ }^{*} \mathrm{p}<0,05 ;{ }^{* *} \mathrm{p}<0,01$

Tabla 4. Medias de las puntuaciones de cada grupo (cluster) de sujetos en las escalas de personalidad del MCMI-Il y en los síndromes clínicos y valor del estadístico t para muestras independientes

\begin{tabular}{|c|c|c|c|}
\hline \multirow[t]{2}{*}{ Personalidad } & Cluster $1(\mathrm{~N}=99)$ & Cluster $2(\mathrm{~N}=135)$ & \\
\hline & Obsesivo compulsivo & Antisocial & $\mathrm{t}$ \\
\hline Dependiente & 60,97 & 60,20 & 0,22 \\
\hline Obersivo-Compulsivo & 72,44 & 58,01 & $4,96^{*}$ \\
\hline Antisocial & 31,89 & 70,44 & $-14,50^{*}$ \\
\hline Narcisista & 38,99 & 63,39 & $-7,78^{*}$ \\
\hline Agresivo-Sádico & 31,86 & 64,51 & $-10,67^{*}$ \\
\hline Pasivo-Agresivo & 19,64 & 69,81 & $19,56^{*}$ \\
\hline Histriónica & 45,83 & 65,38 & $-6,44^{*}$ \\
\hline Autodestructivo & 34,63 & 69,07 & $-12,33^{*}$ \\
\hline Fóbica & 24,36 & 63,78 & $-14,18^{*}$ \\
\hline Esquizoide & 54,39 & 63,95 & $-3,45^{*}$ \\
\hline Paranoide & 49,26 & 68,33 & $-7,04^{*}$ \\
\hline Limite & 20,97 & 62,78 & $-18,33^{*}$ \\
\hline Esquizotípico & 31,40 & 60,51 & $-11,52^{*}$ \\
\hline \multicolumn{4}{|l|}{ Síndromes clínicos } \\
\hline Abuso de alcohol & 41,66 & 65,79 & $-9,32^{*}$ \\
\hline Abuso de drogas & 30,95 & 63,91 & $-15,98^{*}$ \\
\hline Ansiedad & 29,89 & 52,61 & $-6,28^{*}$ \\
\hline Trastorno delirante & 51,56 & 66,49 & $-5,72^{*}$ \\
\hline Hipomanía & 38 & 61,85 & $-9,27^{*}$ \\
\hline Pensamiento psicótico & 30,74 & 62,42 & $-11,66^{*}$ \\
\hline Neurosis depresiva & 23,58 & 41,66 & $-5,16^{*}$ \\
\hline Histeriforme & 32,44 & 50,78 & $-5,56^{*}$ \\
\hline Depresión mayor & 23,76 & 53,89 & $-9,87^{*}$ \\
\hline
\end{tabular}

${ }^{*} p<0,05$

tuaciones más altas en los patrones obsesivo-compulsivo, pasivo-agresivo y dependiente, pudiendo ser identificado como "grupo obsesivo-compulsivo". El segundo muestra puntuaciones más altas en el patrón antisocial y, en general, en todos los considerados de mayor gravedad (agresivo-sádico, autodestructivo, límite y esquizotípico). Este grupo se identifica como "grupo antisocial".

Finalmente, para analizar las diferencias entre los dos grupos en los síndromes clínicos, al realizar la prueba $t$ para muestras independientes se obtienen los resultados que 
se presentan en la Tabla 4. El grupo antisocial muestra, de forma estadísticamente significativa, puntuaciones más altas en todos los sindromes clínicos, siendo cercanas a la puntuación clínica en abuso de alcohol, abuso de drogas, pensamiento psicótico y trastorno delirante.

\section{DISCUSIÓN}

Las conclusiones principales del estudio contribuyen a describir, de forma empírica, los patrones de personalidad y los síndromes clínicos que caracterizan a un grupo de jugadores patológicos. Asimismo los resultados aportan la existencia de dos perfiles de personalidad distintos, en el que están representados todos los sujetos, y que se relacionan de manera desigual con los síndromes clínicos.

Los patrones de personalidad de mayor frecuencia fueron dependiente, obsesivo-compulsivo, antisocial y narcisista, relacionados cada uno con los diferentes síndromes clínicos.

En relación al patrón de personalidad dependiente, se encontró comorbilidad en un $29,7 \%$ de los jugadores. En el estudio de Blaszcynski y Steel (1998), hallaron que un $48,8 \%$ cumplía criterios para el trastorno dependiente de la personalidad y, en menor medida, el estudio de Desai y Potenza (2008) mencionan un porcentaje del 0,8\%. Por otra parte, López-Durán et al., (2007) sugieren que la personalidad dependiente es la que obtiene una mejor evolución en el ámbito de las adicciones, al ser comparada con los demás patrones de personalidad.

Este patrón de la personalidad, correlaciona en este estudio significativamente con los síndromes clínicos de la ansiedad, histeriforme e hipomanía. Desde la perspectiva de Millon (2006), las personas con un patrón de personalidad dependiente, podrían experimentar la ansiedad relacionada con la posibilidad del afrontamiento de nuevas situaciones y la responsabilidad personal. Asimismo, la ansiedad podría generar un estado de ánimo irritable, lo que se relaciona con la presencia del sindrome clínico de la hipomanía en este cuadro. En cuanto al síndrome clínico histeriforme, podría adoptar la manera de un afrontamiento indirecto, expresándose físicamente a través de somatizaciones.

En relación al patrón de personalidad obsesivo-compulsiva el porcentaje es del 29,3\%. Resultados coherentes con la investigación de Black y Moyer (1998), que lo consideran uno de los trastornos de personalidad más diagnosticado en los jugadores patológicos. Bagby et al., (2008), encontraron en su estudio un porcentaje del 64\%. Por otra parte, Henderson (2004), administra el MCMI-II a una muestra de pacientes psiquiátricos ambulatorios con y sin problemas de juego, y llega a la conclusión de que este patrón de personalidad está significativamente relacionado con los problemas del juego. Este patrón obsesivo-compulsivo se ha relacionado única y moderadamente con el síndrome clínico histeriforme, lo que es congruente con la tensión, inquietud e hipersensibilidad a cualquier ambiente que se manifestaría de forma física a través del agotamiento y las respectivas quejas somáticas (Millon, 2006).
En los resultados de este estudio, se obtuvo un alto porcentaje de jugadores evaluados con un trastorno de personalidad antisocial (14,6\%); cifra en consonancia con estudios anteriores (Cunningham-WIliams et al., 1998; CunninghamWilliams et al., 2000; Ibáñez et al., 2001; Pietrzak y Petry, 2005). Para algunos autores es importante la comorbilidad de la personalidad antisocial con el consumo de alcohol y drogas (Cunningham-Williams et al., 2000; Pietrzak y Petry, 2005) Los resultados que se han obtenido concuerdan con esta idea, en este estudio los jugadores que han puntuado alto para patrón de personalidad antisocial obtienen una alta relación con el abuso de drogas y moderada relación con el abuso de alcohol. Según Millon (2006), el consumo de sustancias en estas personalidades disminuye o distrae los efectos negativos residuales, como la ansiedad y la depresión. En cuanto a la relación del síndrome clínico de trastorno delirante y del pensamiento psicótico con el patrón antisocial de personalidad, se podría sugerir que al encontrarse relacionado con el abuso de alcohol y drogas, la sintomatología psicótica podría ser consecuencia de los abusos de sustancias.

Por otra parte, y probablemente muy relacionado con el antisocial, el trastorno de personalidad narcisista en el presente estudio ha obtenido un alto porcentaje $(23,6 \%)$. Algunos estudios (Bagby et al., 2008; Blaszczynski y Steel, 1998; Fernández-Montalvo y Echeburúa, 2006; Kruedelbach et al, 2006; Salazar-Fraile, Ripoll-Alandes y Bobes, 2010), sugieren que este trastorno se relaciona altamente con adicciones como el juego patológico. En cuanto a la relación que obtiene el patrón de personalidad narcisista y el síndrome clínico histeriforme se podría deber a que se trata de una vía más fácil para admitir el malestar, ya que las somatizaciones permiten manifestarse de forma que no resulte tan amenazante para el autoconcepto. Respecto a la relación con el síndrome clínico de hipomanía, Millon (2006) refiere que la personalidad narcisista le es más fácil tener un estado eufórico de la hipomanía, que tener un estado depresivo, ya que éste último le desequilibraría la seguridad que tiene de sí mismo. La relación que presenta con el consumo de drogas, podría situar al jugador al margen de la realidad, encontrándose con una falsa autoestima y seguridad personal, sin conciencia de los aspectos negativos de su situación. Al correlacionar con el pensamiento psicótico y el trastorno delirante sugiere la distorsión de la realidad.

En este estudio, hemos obtenido tres síndromes clínicos de mayor prevalencia. En primer lugar el abuso de alcohol con un 24,5\%, resultados concordantes con diferentes estudios (Black y Moyer, 1998; Cunningham-Williams et al., 1998; Llinares et al., 2003; Kruedelbach et al., 2006; Petry, 2001). Las probabilidades de un diagnóstico de dependencia al alcohol es de dos a cuatro veces mayor entre las personas con diagnóstico de juego patológico, que entre los personas sin problemas de juego (Slutske et al., 2005). En el estudio de French, Maclean y Ettner (2008), manifiestan que esta relación, juego patológico y alcohol, podrían ser actividades complementarias.

En cuanto al abuso de drogas, se obtiene un $16 \%$ de frecuencia en este estudio. El resultado es coherente con el estudio de Fernández-Alba (2004), donde se muestra un 34\% 
de jugadores menores de 30 años de edad que presentan consumo de sustancias ilícitas.

Por otra parte y con una leve diferencia, el síndrome clínico de la ansiedad es observado en 15,6\% de los jugadores. Llinares et al., (2003), sugieren que los altos niveles de ansiedad en los jugadores patológicos, se deben principalmente a la toma de conciencia del problema, a personas que en tratamiento aún continúan jugando y desean lograr la abstinencia.

Siguiendo a Millon se pensó que el patrón de personalidad patológico rara vez se observaría en estado puro (2006), esto es representado en el sujeto en una sola escala del cuestionario. Más bien se trataria de un prototipo o perfil de personalidad en el que se incluyeran, en mayor o menor medida, rasgos de distintos patrones de personalidad. En este estudio se han identificado dos perfiles claramente distintos: un perfil "obsesivo-compulsivo" que incluiría rasgos de escalas de menor gravedad (obsesivo-compulsivos, dependientes) y que muestra puntuaciones bajas en las escalas de síndromes clínicos. Se trataría de un perfil de bajo conflicto y probablemente de buen pronóstico terapéutico. Sin embargo, se aprecia un segundo patrón que denominamos "antisocial" con rasgos de las escalas que indican mayor patologia de personalidad (antisocial, límite, esquizotípico, pasivo-agresivo, agresivo-sádico y autodestructivo) y que, a su vez, se relaciona con las escalas de abuso de drogas, alcohol y escalas cercanas a la psicosis. Esta conclusión creemos podría explicar la diferencia entre los distintos estudios cuando solo se analizan las escalas de manera independiente. Podriamos así aunar conclusiones de autores que presentan distintos trastornos de mayor frecuencia en sus estudios y que podrian realmente estar coexistiendo en las mismas personas (Bagby et al., 2008; Blaszcynski y Steel (1998), Blaszczynski, Steel y McConaghy 1997; Fernández-Montalvo y Echeburúa, 2006; Pietrzak y Petry, 2005; Kruedelbach et al, 2006). En este sentido Blaszczynski y Steel (1998), cuando analizan la coexistencia de trastornos de personalidad en una misma persona obtienen una media de 4,7 trastornos de personalidad para una muestra de 80 sujetos utilizando evaluaciones de autoinforme, y Fernández-Montalvo y Echeburúa (2006) una media de 2,2 trastornos de personalidad, para una muestra de 50 sujetos y con el mismo instrumento que se ha utilizado en este estudio.

En sintesis, un factor importante en los resultados del grupo de jugadores patológicos es la presencia alta de comorbilidad con patrones clínicos de personalidad y con sindromes clínicos.

El juego patológico representa un desorden, que podría emerger en ausencia de otra comorbilidad y causar consecuencias psicopatológicas cualesquiera como desorden secundario, subordinado a un comportamiento disfuncional. Pero también el juego patológico, podría existir como consecuencia a otra condición previa como son patrones patológicos de personalidad (Blaszcynski y Steel, 1998; Black y Moyer, 1998; Echeburúa 2005; 2006; Fernández-Montalvo y Echeburúa 2006; Henderson, 2004; Shaffer y Korn, 2002; Sherrer et al., 2005).
El interés por tanto de este estudio, es aportar mayor evidencia empírica en este sentido en una muestra amplia de personas con problemas de juego patológico, y conocer desde una perspectiva integradora, las diferentes manifestaciones que conforman el cuadro clínico del jugador patológico para asi poder lograr una mayor precisión en el diseño del tratamiento y rehabilitación del jugador patológico. La aportación de dos perfiles clínicos muy distintos puede ayudar a los clínicos a la identificación y valoración de un tratamiento diferencial.

Una de las limitaciones de este estudio podría ser la utilización del cuestionario MCMI-II, habiendose adaptado en la actualidad la siguiente versión del autor (MCMI-III). Esto se podría relacionar con las críticas de falsos positivos de este instrumento (Besteiro, Lemos, Muñiz, Garcia y Álvarez, 2006; Winberg y Vilalta, 2009) que podrían sugerir una dificultad para diferenciar la dualidad real de los resultados. El motivo principal es el haber iniciado el estudio antes de la adaptación del MCMI-III en la lengua castellana y entender necesario recoger todos los datos con la misma adaptación. Otra limitación es que se trata de un estudio correlacional; esto es así por tratarse de una de las etapas de la investigación que tendrá como objetivo posterior la predicción en la rehabilitación de los jugadores patológicos.

\section{AGRADECIMIENTOS}

Expresamos nuestro agradecimiento a AMALAJER, Asociación Malagueña de Jugadores en Rehabilitación, que ha facilitado todo lo necesario para llevar a cabo este estudio y a todas aquellas personas que sufren como consecuencia del juego patológico y que han prestado su importante colaboración.

\section{REFERENCIAS}

American Psychiatric Association (APA) (1980). Diagnostic and Statistical Manual of Mental Disorders, $3^{\text {rd }}$ edition (DSM-III). Washington, D.C.: Author.

American Psychiatric Association (APA) (2000). Diagnostic and Statistical Manual of Mental Disorders, $4^{\text {th }}$ edition (DSM-IV). Washington, D.C.: Author.

Avila-Espada, A., Jiménez Gómez, F. y cols. (1999). Adaptación y baremación al español del Inventario Clínico Multiaxial de Millon-II (MCMI-II). Madrid: TEA, Ediciones.

Bagby, M., Vachon, D., Bulmash, E. y Quilty, L. (2008). Personality disorders and pathological gambling: A review and e-examination of prevalence rates. Journal of Personality Disorders, 22, 191-207.

Bahamón, M. (2006). Juego patológico: revisión de tema. Revista Colombiana de Psiquiatría, 35, 380-399.

Besteiro, J., Lemos., S., Muñiz, J., García, E. y Álvarez, M. (2006). Dimensiones de los trastornos de personalidad en MCMI-II. 
International Journal of Clinical and Health Psychology, 7, 295306.

Black, D. y Moyer, T. (1998). Clinical features and psychiatric comorbidity of subjects with pathological gambling behavior. Psychiatric Services, 49, 1434-1439.

Blaszczynski, A., Steel, Z. y McConaghy, N. (1997). Impulsivity in pathological gambling: the antisocial impulsivity. Addiction, 92, 75-87.

Blaszczynski, A. y Steel, Z. (1998). Personality disorders among pathological gamblers. Journal of Gambling Studies, 14, 51-71.

Cardenal, V., Sánchez-López, M. y Ortiz-Tallo, M. (2007). Los trastornos de personalidad según el modelo de Millon: una propuesta integradora. Clínica y Salud, 18, 305-324.

Cunningham-Williams, R., Cottler, L., Compton, W. y Spitznagel, E. (1998). Taking chances: problem gamblers and mental health disorders: results from the St. Louis epidemiologic catchment area Study. American Journal of Public Health, 88, 1093-1096.

Cunningham-Williams, R., Cottler, L., Compton, W., Spitznagel, E. y Ben-Abdallah, A. (2000). Problem gambling and comorbid psychiatric and substance use disorders among drug users recruited from drug treatment and community settings. Journal of Gambling Studies, 16, 347-75.

Desai, R. y Potenza, M. (2008). Gender differences in the associations between past-year gambling problems and psychiatric disorders. Social Psychiatry and Psychiatric Epidemiology, 43, 173-183.

Durdle, H. y Stewart, S. (2007). Should pathological gambling be considered an obsessive-compulsive spectrum condition? En J. S. Abramowitz, D. McKay, y S. Taylor (Eds.), Obsessive-compulsive disorder: Subtypes and Spectrum Conditions (pp. 160-176). New York, NY: Elsevier.

Echeburúa, E. (2005). Retos del futuro en el tratamiento del juego patológico. Adicciones, 17, 11-16.

Echeburúa, E. (Comp.) (2006). Nuevas fronteras en el estudio del juego patológico. Madrid: Fundación Ramón Arces.

El-Guebaly, N., Patten, S., Shawn, C., Williams, J., Beck, C., Maxwell, C. y Wang, J. (2006). Epidemiological associations between gambling behavior, substance use \& mood and anxiety disorders. Journal of Gambling Studies, 2, 275-287.

Estévez, A. y Calvete, E. (2007). Esquemas cognitivos en personas con conducta de juego patológico y su relación con experiencias de crianza. Clínica y Salud, 18, 23-43.

Fernández-Alba, A. (2004). Caracteristicas clínicas y comorbilidad psiquiátrica en jugadores patológicos de maquinas recreativas con premio, en tratamiento: Estudio de la población menor de 30 años. Adicciones, 16, 7-18.

Fernández-Montalvo, J. y Echeburúa, E. (2006). Juego patológico y trastornos de personalidad: un estudio piloto con el MCMI-II. Psicothema, 18, 453-458.

French, M., Maclean, J. y Ettner, S. (2008). Drinkers and bettors: Investigating the complementarity of alcohol consumption and problem gambling. Drug and Alcohol Dependence, 96, 155-164.
Goodyear-Smith, F., Arroll, B., Kerse, N., Sullivan, S., Coupe, N., Tse, S., (2007). Primary care patients reporting concerns about their gambling frequently have other co-occurring lifestyle and mental health issues. BMC Family Practice, 7, 25.

Henderson, M. (2004). Psychological correlates of comorbid gambling in psychiatric outpatients: A pilot study. Substance Use \& Misuse, 39, 1341-1352.

Ibáñez, A., Blanco, C., Donahue, E., Lesieur, H., Pérez, I., Fernández, J. y Sáiz, J. (2001). Psychiatric comorbidity in pathological gamblers seeking treatment. American Journal of Psychiatry, 158, 1.7331.735 .

Kim, W. y Grant, J. (2001). Personality dimensions in pathological gambling disorder and obsessive-compulsive disorder. Psychiatry Research, 104, 205-212.

Krishnan, M. y Orford, J. (2002). Gambling and the family: From the stress-coping-support perspective. International Gambling Studies, 2, 61-83.

Kruedelbach, N., Walker, H., Chapman, H., Haro, G., Mateu, C. y Leal, C. (2006). Comorbilidad de trastornos con pérdida de control de impulsos: ludopatía, adicciones y trastornos de la personalidad. Actas Españolas de Psiquiatría, 34, 76-82.

Labrador, F. (2008). Abordaje psicológico del diagnóstico y el tratamiento del juego patológico. Jano, 1681, 29-32.

Llinares, M., Santos, P., Camacho, I., Albiach, C. y Palau, C. (2003). Variables psicológicas y adictivas en una muestra de jugadores patológicos. Anales de Psicología, 19, 75-80.

López-Duran, A., Becoña, E., Lloves, M., Moneo, A., Vieitez, I., Cancelo, J. et al. (2007). Evolución del tratamiento y consumo en personas con dependencia a la cocaina, a los doce meses, en función de la psicopatología asociada y el funcionamiento cotidiano. Adicciones, 19, 103-109.

Melville, K., Casey, L. y Kavanagh, D. (2007). Psychological treatment dropout among pathological gamblers. Clinical Psychology Review, 27, 944-58.

Millon, T. (1987). MCMI-II. Millon Clinical Multiaxial Inventory-II. National Computer Systems, Inc. Minneapolis.

Millon, T. (2006). Trastornos de personalidad en la vida moderna $\left(2^{a}\right.$ ed.). Barcelona: Masson.

Ortiz-Tallo, M., Cardenal, V., Ferragut, M. y Cerezo, Ma.V. (2011). Personalidad y sindromes clínicos. Un estudio con el MCMI basado en una muestra española. Revista de Psicopatología y Psicología Clínica, 16, 49-59.

Pedrero Pérez, E.J. (2009). Dimensiones de los trastornos de personalidad en el MCMI-II en adictos a sustancias en tratamiento. Adicciones 21, 29-37.

Pietrzak, R. y Petry, N. (2005). Antisocial personality disorder is associated with increased severity of gambling, medical, drug and psychiatric problems among treatment-seeking pathological gamblers. Addiction, 100, 1183-1193.

Rodriguez-Jimenez, R., Avila, C., Jimenez-Arriero, M., Ponce, G., Monasor, R., Jimenez, M. et al. (2006) Impulsivity and sustained 
attention in pathological gamblers: Influence of childhood ADHD history. Journal of Gambling Studies, 22, 451-461.

Sacco, P., Cunningham-Williams, R., Ostmann, E. y Spitznagel, E. (2007). The association between gambling pathology and personality disorders. Journal of Psychiatric Research, 42, 11221130.

Salazar-Fraile, J., Ripoll-Alandes, C. y Bobes. J. (2010). Narcisismo manifiesto, narcisismo encubierto y trastornos de personalidad en una Unidad de Conductas Adictivas: validez predictiva de respuesta a tratamiento. Adicciones 22, 107-112.

Sherrer, J., Xian, H., Shah, K., Volberg, R., Slutske, W. y Eisen, S (2005). Effect of genes, environment, and lifetime co-occurring disorders on health-related quality of life in problem and pathological gamblers. Archives of General Psychiatry, 62, 677683.

Slutske, W., Caspi, A., Moffit, T. y Poulton, R. (2005). Personality and problem gambling: A prospective study of a birth cohort of young adults. Archives of General Psychiatry, 62, 769-775.

Stewart, S. y Kushner, M. (2003). Recent research on the co-morbidity of alcoholism and pathological gambling. Alcoholism: Clinical and Experimental Research, 27, 285-291.

Winberg, M. y Vilalta, R. (2009). Evaluación de trastornos de personalidad mediante el inventario clínico multiaxial (MCMI-II) en una muestra forense. Psicothema, 21, 610-614. 
